\title{
L'Incertitude de Newton à Heisenberg Un Nouveau Paradigme pour l'Economique et ses Effets sur la Notion de Risque et sur le Rôle Economique des Activités des Assurances *
}

\author{
par Orio Giarini **
}

\section{Introduction}

Cet exposé traite principalement des questions suivantes :

- l'idée que les sciences économiques sont encore principalement un produit intellectuel de la révolution industrielle « classique » : si nous vivons réellement dans une " société post-industrielle », ou "société des services », certains paradigmes fondamentaux notamment la notion de valeur - doivent être radicalement reconsidérés (section 2);

- cette remise en question des notions économiques de base, comme toutes les remises en question, trouve sa justification dans un contexte culturel élargi, notamment en ce qui concerne la définition de la science et la valeur que lui confère la société en tant que moyen d'approfondissement des connaissances; on soutient généralement que l'économie est encore largement liée à la définition de la science qui a prévalu jusqu'au début du $20^{\mathrm{e}}$ siècle (positiviste, Newtonienne). En tant que telle, elle favorise une notion statique du temps et de l'espace, et des modèles basés sur des hypothèses de certitude.

L'activité des assurances, analysée comme il convient d'un point de vue économique, nécessite une véritable dimension espace/temps, et le remplacement du principe de la certitude par celui de l'incertitude. L'intégration des activités des assurances à une théorie économique globale coïncide avec l'incorporation et l'adaptation de notions économiques de base à la pensée spécifique contemporaine (section 3) ;

- le succès futur des sciences économiques dépendra dans une large mesure de la possibilité d'inclure l'incertitude (et le temps réel) dans les modèles, les théories et la

* The English version of this text has been published in "Geld, Banken und Versicherungen ", by VVW Karlsruhe; Editors: H. Göppl and R. Henn, 1983, pp. 903-922.

** Institut Universitaire d'Etudes Européennes, Genève. 
recherche empirique, suivant ainsi le paradigme d'Heisenberg. Cela permettrait de confirmer à l'assurance une plus grande importance au sein de l'activité économique (section 4).

\section{La science économique comme théorie du processus d'industrialisation}

On admet généralement que la science économique a pris sa forme définitive en tant que science ou discipline spécifique en 1776, avec la publication par Adam Smith de " La Richesse des Nations ». Comme l'a fait remarquer Alfred Marshall, "sa principale réalisation fut de trouver dans la théorie de la valeur un centre commun qui a donné son unité à la science économique » ([10], p. 627). Cette notion de valeur rejoignait à la fois la réalité historique et les principes ou paradigmes culturels de l'Europe de son temps, dont l'événement principal fut la naissance d'une longue période de développement économique connue sous le nom de révolution industrielle, et basée sur trois facteurs interdépendants :

a) le développement du mode de production industriel qui devenait, pour la première fois dans l'histoire, le premier facteur déterminant de la production de bien-être et la base du pouvoir ;

b) le développement de nouvelles technologies et des moyens techniques aptes à les utiliser, par le processus de la concentration (économies d'échelle) et de la spécialisation ;

c) la monétarisation de l'économie dans une mesure jamais atteinte auparavant. Ivan Illitch [8] a établi qu'au cours des $16^{\mathrm{e}}$ et $17^{\mathrm{e}}$ siècles en Europe, $1 \%$ seulement de la durée moyenne de vie des gens était consacré à un emploi rémunéré. De nos jours, ce pourcentage atteindrait $16 \%$. Cela revient à dire que si l'argent a toujours existé, ce n'est que depuis la révolution industrielle que son volume et sa circulation ont un réel impact sur l'économie dans son ensemble.

Il est important de souligner le fait que la science économique elle-même découle de l'avènement de la révolution industrielle. Pour Adam Smith, il est clair que la valeur de production qui accroît le bien-être est celle qui émane de ce que nous appelons à présent industrialisation ou production de type industriel; par contre, ce que nous appelons le tertiaire, c'est-à-dire les activités de service (médecins, avocats, services financiers, assurances, etc.), ne sont pas - pour Adam Smith - que des activités « respectables », mais économiquement improductives '.

En d'autres termes, la spécialisation de l'économie est telle qu'elle a en fait tendance à se concentrer dès le départ sur le processus d'industrialisation. La notion de valeur elle-même effectue une sélection entre ce qui sera ou non inclus dans les modèles analytiques.

Mais cette méthode de sélection ne dépend pas seulement des préférences personnelles: si cela avait été le cas, elle aurait changé après Adam Smith. Tout au

1 « Le travail de certaines de plus respectables corporations de nos sociétés est, comme celui des domestiques de plus bas niveau, improductif ... les officiers de justice ... l'armée ... la sécurité sociale ... les gens d'église, avocats, médecins, gens de lettres de tout genre ... bouffons ... chanteurs d'opéra ... » (A. Smith [16], pp. 430-443). 
contraire, cette sélection s'est faite de plus en plus précise avec le temps, pour des raisons nombreuses et profondes, certaines d'entre elles provenant des fondements mêmes de la philosophie européenne.

Donner une définition verbale ne suffisait pas - et ne suffit toujours pas - à créer une nouvelle discipline de façon «scientifique». Pour pouvoir être qualifiée de "scientifique " une théorie se doit d'être vérifiable et les "faits " doivent être mesurés. C'est en répondant à cette exigence qu'Adam Smith a jeté les bases définitives de la science économique: la notion de valeur quantifiée à travers un système de prix. Le système de marché (la « main invisible »), par la loi de l'offre et de la demande, établit un prix afin de rémunérer la valeur produite par l'homme.

Un certain nombre de commentaires importants doivent être faits ici :

- l'importance de la «main invisible » réside avant tout dans le fait qu'elle suggère que le prix est une mesure "objective". C'est son aspect "objectif» qui compte réellement et ouvre la porte aux prétentions "scientifiques" des économistes de toutes tendances politiques ;

- grâce à l'outil que représentent les valeurs quantifiables (les prix), les économistes ont espéré devenir aussi "scientifiques" que les chercheurs des sciences naturelles, qui peuvent, dans une très large mesure, déterminer les systèmes ou les modèles qu'ils analysent par la mesure de facteurs spécifiques quantifiables (chaleur, vitesse, poids, inertie, résistance, longueur d'onde, etc.) ${ }^{2}$;

- si les prix sont la clé de l'analyse économique, les sciences économiques doivent avant tout se concentrer sur la valeur monétarisée de la production et de la consommation. Bien que les économistes classiques continuent à discuter sur des valeurs non-monétarisées ils abandonneront peu à peu cette notion, d'une part parce qu'ils travaillent de plus en plus selon l'hypothèse que ce qui est "libre " n'est pas rare, et le considèrent en dehors des frontières de l'économie (ce qui nous paraît incorrect), et d'autre part parce qu'ils ne voient pas comment en donner une mesure exacte (à cause de leurs principes implicitement cartésiens);

- il est évident que la notion de valeurs monétarisées et de prix est obligatoirement liée à la situation d'une économie industrielle en expansion où les échanges et la production suivent le schéma de la monétarisation. Comme nous l'avons déjà souligné, c'est précisément au moment de la naissance et du développement de la révolution industrielle que le système de la monétarisation s'est largement répandu. On devrait relever ici la grande confusion qui règne généralement dans ce qu'on écrit sur la monnaie (ses origines et son utilisation), et sur la question de la transition des activités économiques vers un système monétarisé. La question clé qui se pose aujourd'hui est celle de l'équilibre entre ces deux systèmes ${ }^{3}$;

2 En fait, la plupart des économistes considèrent comme définitive la notion d'une science fondamentalement positiviste, et ne vont pas au-delà des idées de Newton. Voir Mayer [11]: Cet article a été rédigé sans qu'il y ait la moindre référence explicite à ce que les sciences de la nature représentent aujourd'hui, la physique en particulier. Se référer au contraire à Weisskopf [17], selon lequel «la fiction de la «main invisible » était et reste une technique de tendance newtonienne afin de se protéger contre le caractère mystérieux, indéterminé et incertain de l'action sociale ».

3 Voir Giarini [4], chap. 3. 
- la raison d'une croissance décisive de la monétarisation pendant la révolution industrielle est étroitement liée à un autre facteur fondamental : le développement de la technologie moderne. Plus la technologie sera "moderne », plus elle sera coûteuse, c'est-à-dire qu'elle nécessitera davantage de capital (monnaie accumulée). Et afin de pouvoir accumuler efficacement la monnaie, le système économique se doit d'être de plus en plus monétarisé. Au dix-huitième siècle les besoins en capital étaient, en Angleterre, comme l'a souligné Adam Smith, beaucoup plus élevés que jamais auparavant (jusqu'à 5-6\% des ventes...). Ce pourcentage atteignit $12-15 \%$ au cours du siècle dernier, et 25 et $30 \%$ de nos jours.

L'industrialisation est donc essentiellement l'histoire du développement technologique et des besoins en capital, de la spécialisation et de la monétarisation. Une société qui n'est pas portée vers la monétarisation a plus qu'une autre des difficultés à utiliser et bénéficier de la technologie moderne, comme cela s'est passé en Europe ;

- Adam Smith a par conséquent, en concentrant son attention sur le développement de l'industrialisation, et en fondant son analyse économique sur la notion de prix, atteint le cœur même de l'histoire économique de ces deux derniers siècles ;

- On doit souligner aussi le fait que tous ce développement de la révolution industrielle classique cadre assez bien avec la méthode et la philosophie cartésienne, qui apparaît par conséquent aussi comme un instrument de légitimisation culturelle et idéologique. Richesse et bien-être augmenteront pendant deux siècles dans des proportions sans précédent, grâce surtout à l'existence d'un cycle long de rendements croissants de la technologie ${ }^{4}$

Dans ce contexte il faudrait signaler que la priorité donnée à l'industrialisation ressort clairement de la division de l'activité économique (monétarisée), encore de nos jours, en secteurs primaire (agricole), industriel et tertiaire : dans cette classification, c'est le secteur industriel qui joue le rôle moteur essentiel.

L'un des principaux points à comprendre lorsque l'on parle de changements structurels dans la situation économique actuelle est que la réalité de l'économie « post-industrielle» ou "des services" ne provient pas seulement d'une évolution du secteur tertiaire. La clé de ce phénomène ne réside pas exclusivement dans la croissance de ce dernier secteur aux dépens des deux autres. La subdivision n'est plus verticale : elle est horizontale. D'une part, l'agriculture et les services utilisent du matériel et des biens d'équipement typiques des activités industrialisées. D'autre part, les secteurs industrialisés eux-mêmes font une utilisation accrue de fonctions "de service ", qui sont souvent celles que les économistes classiques qualifient d'« improductives » (bien qu'elles occupent toujours la majeure partie de la main-d'œuvre) ${ }^{5}$, à l'intérieur même des activités de production.

Il est par conséquent erroné et source de confusion d'une part d'accepter l'idée que le secteur des services revêt une grande importance dans notre société, et d'autre part, de continuer à classifier ce phénomène avec des méthodes qui correspondent à des

${ }^{4}$ Voir Giarini et Loubergé [5].

5 Voir le second chapitre in Giarini [4]. 
circonstances largement dépassées, sans vérifier l'évolution des fonctions de service dans les processus de fabrication.

La raison pour laquelle on applique encore toujours d'anciennes classifications tout en admettant qu'elles ne correspondent plus à la réalité économique actuelle, c'est que peu de travaux ont porté sur les notions de base qui sont à l'origine de ces mêmes classifications ${ }^{6}$.

\section{La théorie économique et ses paradigmes scientifiques de référence: le cas de l'assurance}

Notre thèse est donc que l'« économique ", qui s'est développée en tant que discipline ou science depuis Adam Smith, est essentiellement la théorie du processus d'industrialisation, dans la mesure où ce processus représente la principale contribution à l'accroissement de la richesse des nations. De ce point de vue, tous les phénomènes qui ne font pas partie intégrante du processus industriel de production sont secondaires, ou considérés comme tels en pratique ou en théorie. Ceci est vrai même si l'histoire de la pensée économique a beaucoup évolué depuis Adam Smith, et même si elle embrasse de plus vastes horizons. En fait, même si, dans de nombreux cas, la science économique s'est efforcée d'aller au-delà du processus d'industrialisation lui-même, elle a toujours considéré ce dernier comme étant la référence clé pour tout autre type d'activité. Par le système des prix fictifs, ou par l'analyse de la valeur des services, la science économique a toujours tâché de ramener toute analyse au " paradigme " industriel comme référence de base. En d'autres termes, au fur et à mesure que l'importance des services augmente, la "science économique» est de moins en moins représentative de l'«économie», c'est-à-dire de la manière dont bien-être et richesses sont actuellement effectivement produits et distribués. Cet état de fait trouve ses racines dans une vérification insuffisante de l'adéquation des théories économiques contemporaines aux conditions épistémologiques actuelles. En particulier, la pensée économique est encore excessivement liée aux concepts traditionnels cartésiens (et newtoniens) de la science.

La pensée cartésienne d'une part s'avère toujours efficace dans des conditions où l'industrialisation est la première priorité et est le meilleur moyen d'organiser la richesse et le bien-être, et d'autre part soulève une série de difficultés pratiques et méthodologiques à partir du moment où l'économie dépend de plus en plus des services et de l'environnement. Isoler les facteurs économiques sur la base de leur monétarisation est une méthode qui présente de nos jours de plus en plus de faiblesses. Afin de clarifier ce point, revenons tout d'abord à la notion de science telle qu'elle est perçue par les économistes.

\footnotetext{
${ }^{6}$ Nous pensons également que, bien que la notion de valeur ne soit plus liée à production (comme dans la pensée économique classique), mais plutôt aujourd'hui orientée vers les aspects subjectifs de la demande (comme pour les économistes néo-classiques), les hypothèses industrialisantes cartésiennes sous-jacentes du modèle économique général demeurent, en pratique, inchangées. Plus encore, la théorie subjective de la valeur rend la science économique de plus en plus inapte, à maints égards, à définir et organiser les facteurs de production. Ce qui fait fortement défaut aujourd'hui est une nouvelle notion de valeur post-industrielle, du côté de l'offre.
} 
Au $19^{e}$ siècle, il était courant de penser que la méthode cartésienne ou newtonienne ${ }^{7}$ de recherche scientifique consistait avant tout à définir clairement une situation ou un problème, d'identifier et de mesurer tous ses constituants, comme si cette situation ou ce problème pouvait être parfaitement et totalement défini en tant que système isolé (ou en assumant que rien de ce qui en avait été laissé de côté n'avait une influence appréciable sur le système observé). C'est de cette façon qu'une molécule d'eau peut être isolée et étudiée ou que Newton a donné une vision claire de la mécanique du ciel. De la même façon les économistes ont espéré pouvoir fournir des modèles de réalité scientifiquement définis et déterminés. L'idée sous-jacente est que la réalité observée est essentiellement «objective». Cette approche présuppose des systèmes simples, et, comme corollaire, la division discrète du temps et de l'espace.

Il est clairement apparu dans les sciences naturelles depuis de nombreuses décennies, que même s'il existe une multitude de réalités que l'on peut étudier à la manière cartésienne, lorsqu'il s'agit de revenir à des définitions fondamentales (telles que: de quoi s'agit-il ?) et à des questions liées à l'« objectivité " (si cela existe) on se trouve dans des systèmes extrêmement complexes et indéterminés ${ }^{8}$.

$\mathrm{Au}$ cours des dernières décennies on a constaté avec surprise qu'alors que les scientifiques «sociaux» de toutes tendances - notamment les économistes s'efforçaient de donner une image "objective» de leur «science", et avançaient souvent que cette science supporterait un jour la comparaison avec les sciences

$7 \mathrm{~W}$. Weisskopf [17] l'affirme clairement: «Le paradigme newtonien, repris par la théorie économique classique et néo-classique, a interprété l'économie conformément au schéma mis au point dans les sciences physiques et mécaniques, et par analogie au système planétaire et aux horloges: un système autonome, clos, géré par des facteurs mutuellement interdépendants, très sélectifs et auto-régulateurs, qui tendent vers un point d'équilibre déterminé et prévisible. Le paradigme de Newton, qui va de pair avec la pensée économique du $18^{\mathrm{e}}$ siècle, présente les événements économiques comme une réalité indépendante de l'observateur. L'observateur est détaché du sujet observé, mais il peut le saisir par le raisonnement. Une réalité objective, soumise aux lois naturelles, peut être appréhendée et comprise par la raison humaine. L'idée de loi naturelle faisait le lien entre le sujet et l'objet, les reliant, malgré leur indépendance réciproque, par le raisonnement «scientifique ».

C'est ainsi que sujets, objets, loi naturelle et raisonnement, tous indépendants les uns des autres, formaient une configuration à quatre unités. Les lois naturelles étaient causales, considérées comme causae efficientes, non comme causae finales; comme forces motrices, non comme motivations et aspirations; non seulement dans la nature matérielle, mais aussi dans le domaine de la vie humaine. Le but de ce courant de pensée était de prédire les événements futurs, et d'arriver à des solutions à tous les niveaux de la réalité. Si toutes les variables, toutes les relations de cause à effet étaient connues, on pourrait comprendre et prédire les événements de l'Univers, de la société, et toutes les actions humaines. La plupart des scientifiques étaient convaincus - et le sont encore dans une large mesure - que des lois inéluctables déterminaient tous les événements et toutes les actions, malgré l'ignorance provisoire qu'en ont les hommes. Ils ne laissaient aucune place à la liberté, au choix, à l'incertain, au mystère. Ce courant de pensée fut repris par les théories économiques classiques et néo-classiques comme fondement des modèles d'équilibre, complétée par des hypothèses fictives comme la connaissance parfaite et la projection idéale, et en remplaçant le temps et les changements par la clause du ceteris paribus. »

${ }^{8}$ Indéterminé au sens d'Heisenberg. Toute la controverse commencée par Einstein avec sa réalité "probable » est une référence importante dans ce cas. 
naturelles les plus «scientifiques», ces dernières ont, entre-temps, renversé la tendance .

La conséquence que cela entraîne pour les sciences économiques et les sciences sociales en général est que leur validité est essentiellement limitée à des faits (historiques) donnés et que l'appellation "validité universelle" (dans le temps et l'espace) n'a qu'un sens limité.

Si ce qu'on vient d'énoncer n'est qu'en partie acceptable, il s'ensuit néanmoins que la science économique aurait intérêt à remettre en question certaines de ses hypothèses de base, surtout la notion de valeur, sur laquelle l'économie se fonde, et ses déterminants historiques et culturels, avec référence surtout aux notions de temps et d'espace ${ }^{10}$.

Dans l'univers cartésien ou newtonien, le temps est soit infini soit spécifique : on peut isoler une période de temps. On peut, de manière statique, examiner la "réalité " comme on le fait d'une photographie, en arrêtant tout mouvement. L'équilibre de l'univers vu par Newton est semblable à l'équilibre entre les courbes d'offre et de demande des économistes: à un moment donné (un moment fixe dans le temps) la

9 Au point qu'un Prix Nobel comme Prigogine aperçoit à présent la possibilité d'une « nouvelle alliance " entre sciences naturelles et sciences humaines. Elles ne sont plus opposées par essence; elles sont plus ou moins indéterminées [4] et [15]. Quant à Weisskopf [17], il définit le paradigme d'Heisenberg de la façon suivante : «Le principe d'incertitude d'Heisenberg (ou d'indétermination) suppose que - en microphysique - l'influence que peut avoir l'observateur sur la position et la vitesse des particules empêche de définir avec exactitude à la fois leur position et leur vitesse. Ainsi, les bases de prévisions précises sont détruites. " Ceci entraîne une vue différente de la réalité : "Il n'existe pas d'estimation causale complète du futur sur la base de notre connaissance du présent. Cela signifie que chaque ... mesure ... crée ... une situation unique, imparfaitement prévisible. " Il tirait la conclusion que « l'homme ne peut observer le cours de la nature sans l'altérer. » Niels Bohr déclarait que «l'homme est à la fois acteur et spectateur du drame de la vie», et Max Born comparait la situation à « une partie de football au cours de laquelle le fait d'assister ... applaudir ou siffler avait une influence ... sur les joueurs et par conséquent sur ce qui est observé ».

«L'homme est un être fini et conditionné. Il est conditionné par son anatomie, sa physiologie, l'histoire de sa vie, son environnement social, et un nombre infini d'autres facteurs. La position du savant ne diffère pas de celle de l'homme ; c'est également un sujet soumis aux mêmes conditions. Il ne peut sortir de lui-même. Son horizon de connaissance est limité par ce qui le conditionne. Dans les limites de ces conditions, l'homme est libre, et il peut les transcender sans limite grâce à sa conscience. Néanmoins, cette connaissance, scientifique ou autre, contient ces conditions sous forme (souvent latente) de postulats. La réalité qu'il perçoit est la vraie réalité aux conditions de son existence. 11 pense et connaît, mais est lui-même un être conditionné.

Cette analyse ontologique contient des idées similaires au principe de l'indétermination en physique et pourrait être appelée le paradigme heisenbergien philosophique. Ce n'est pas par pure coïncidence accidentelle que des idées similaires se soient développées dans des domaines si différents. Elles trouvent leurs racines dans l'esprit de l'époque. Le nouvel environnement des métaphysiques, de la physique, de la politique, est celui de l'incertitude. Le modèle newtonien, dans sa conclusion finale, élève l'homme, observateur objectif, détaché et "scientifique » au niveau d'une déité omnisciente pouvant prédire l'avenir. Au contraire, le modèle d'Heisenberg réduit l'homme à un participant qui ne peut se dégager de la réalité qu'il veut analyser. Cette nouvelle vision du monde fait ressortir la faiblesse et l'incertitude inhérentes à la nature humaine et qui avaient été réprimées et niées par la pensée de Newton." "

C'est le fait de reconnaître que «l'action est la prise de conscience d'un nouveau commencement dont l'aboutissement est incertain " qui rend l'« action » à la fois réelle et possible.

10 Voir dans [4], l'analyse de la société post-industrielle ou des services, chap. 1 et 2. 
situation est telle ou telle. Des forces simples et identifiables déterminent les situations d'équilibre, et chaque état ou situation peut être isolé.

Dans de telles conditions, le comportement relatif des phénoménes dans le temps et l'espace tend à disparaître, ou se trouve représenté dans un contexte statique et par là limitatif.

Il faut relever ici que l'activité de l'industrie de l'assurance représente une situation intéressante, où l'équilibre de l'offre et de la demande (le prix d'une police d'assurance " aujourd'hui » et le coût du dommage « demain " couvert par la police) est une question de temps réel et incertain. La durée (combien de temps) et l'espace (l'univers statistique) doivent être pris en considération.

Il est facile de se rendre compte, dans ce cas particulier, à quel point sont profondément enracinées les causes qui ont jusqu'à présent empêché que l'assurance (et les services en général) ne soit considérée comme aussi importante que les activités de production (ces dernières pouvant être plus aisément ramenées dans un cadre statique, bien que cela soit de moins en moins acceptable à cause des délais d'adaptation de la technologie moderne).

Irving Pfeffer a démontré, entre autres, que l'analyse du risque, et surtout du risque en assurance, nécessite un cadre analytique dynamique, alors que la théorie économique a choisi une méthode d'analyse statique (voir [13] et [14]).

Ainsi, en économie, la notion de valeur est essentiellement statique (valeur donnée à un moment précis dans le temps, notamment au moment de l'échange). Par conséquent, l'image d'un modèle, en économie, qui cherche à être photographique, exclut plusieurs facteurs importants :

- non seulement l'évolution probable, en état d'incertitude, du comportement économique est écartée (comme dans les sciences physiques avant les années 20) au point que la majorité des modèles économiques impliquent une hypothèse de « certitude » (on dit parfois que ceci est fait par souci de «simplification »);

- mais tout comportement économique couvrant un certain laps de temps disparaît : dans le système national de comptabilité de l'assurance, seuls les employés des compagnies d'assurance sont considérés comme faisant partie de la "valeur ajoutée " (ils sont payés « aujourd'hui » comme dans les autres secteurs, c'est-à-dire en pratique tous les mois - ce qui représente une période de temps courte "1). La valeur réelle de l'assurance, l'augmentation de la richesse rendue possible par la possibilité de contrôler des dommages futurs au moyen du système de l'assurance, n'a pas de valeur...

Les économistes ont dû trouver un artifice pour écarter cette contradiction. Ils parlent de "transferts " qu'ils expliquent ainsi : si une maison brûle, l'assurance paiera pour sa reconstruction (si elle était assurée). L'action de construire est une valeur ajoutée

1 Temps statique et temps court ne sont bien entendu pas identiques, mais un « temps court » permet, en pratique, une analyse statique, alors qu'une telle analyse paraît bien difficile sur des phénomènes à long terme. Il en découle la difficulté par les politiques économiques de sortir du court terme, si non par des prétentions à la planification dite souple (très peu efficace) ou rigide (inévitablement irréaliste). 
et apparaîtra en tant que telle dans les statistiques, et en tant que croissance de l'activité industrielle. Il n'est donc pas besoin de comptabiliser l'assurance en tant que valeur, puisqu'elle a principalement pour effet d'organiser des transferts financiers. Ce qui est "productif», c'est le fait de construire, alors que le fait de rendre cette construction possible est, bizarrement, « non productif » : la priorité donnée à l'activité " industrielle » sur le service est nettement une question de préférence, ou - à la limite - d'idéologie.

En fait, dans le cas de la maison qui brûle, ce qui se passe en temps réel et en termes de richesse réelle est le suivant :

- lorsque la maison est construite, elle apparaît comme valeur ajoutée, ce qui est normal et correct ;

- quand elle brûle, la valeur de la richesse réelle diminue (production d'une valeur de diminution, ou valeur ajoutée négative), mais aucune statistique macro-économique n'en tient compte ;

- quand la maison est reconstruite, grâce au fait qu'elle était assurée, le système de comptabilité économique enregistre cette construction comme une nouvelle valeur ajoutée (industrielle). Ce qui pourrait d'ailleurs engendrer l'idée absurde que plus il y aurait de destructions accidentelles plus on aurait de chances d'accroître la valeur ajoutée. Pour corriger cette inexactitude il faudrait au moins tenir compte de la valeur ajoutée négative ;

- reconstruire une maison grâce à une police d'assurance représente une valeur qui pourrait être entièrement imputée à l'activité des assurance. La maison n'aurait peut-être pas été reconstruite sans assurance. Dans ce cas, les équipements industriels sont partie intégrante du mécanisme (ou service) de sécurité engendré par l'assurance.

Un économiste traditionnel dira que l'assurance est payée pour un service, qui est l'organisation du transfert financier (par l'activité des employés d'assurances et du matériel de bureau) et que l'argent alloué à la reconstruction de la maison participe à nouveau à l'activité industrielle, produisant une valeur ajoutée industrielle. Mais cette assertion est visiblement erronée, tout au moins du point de vue de l'acquéreur de l'assurance, étant donné qu'il achète la possibilité de reconstruire sa maison, et non uniquement un service de transfert financier. Et il acquiert cette possibilité auprès d'une compagnie d'assurance, tout comme l'acquéreur d'un produit d'une usine chimique achète aussi les activités de service incorporées dans la fabrication du produit.

En réalité, le problème n'est pas de donner aujourd'hui la priorité aux industries de service sur les productions industrielles, mais de reconnaître l'intégration des deux (et de l'agriculture) à la production de richesse d'une façon différente de celle qui avait cours au temps de la révolution industrielle. Il faut donc insister sur le fait que, dans la pratique, le concept traditionnel de valeur isole et donne la priorité aux activités industrielles propres, alors que ce que l'on propose ici est une nouvelle conception de la valeur correspondant à la société post-industrielle, destinée à faciliter l'adaptation de l'instrument qu'est la pensée économique à la situation économique présente, dans laquelle activités industrielles et de service sont au même niveau.

Lorsque les assurances et toutes les autres activités de service ne représentaient qu'une faible part de l'économie, cela n'avait guère d'effet pratique, surtout alors que l'économie était dans sa phase ascendante de révolution industrielle. Dans de telles 
conditions, l'argumentation présentée ici pourrait être rejetée comme un exercice intellectuel cherchant à couper les cheveux en quatre.

La situation est différente dans un environnement post-industriel où l'on commence à se rendre compte que la richesse et le bien-être dépendent de plus en plus de mécanismes qui rendent tout produit et tout service disponible et accessible, plutôt que de simples activités de production ${ }^{12}$.

L'exemple suivant permettra d'éclaircir cette question des changements de la structure économique :

Après la crise de 1929 , beaucoup de pauvres gens ne pouvaient acheter de quoi manger par manque d'argent. On détruisait aussi certains types d'aliments afin de ne pas inonder le marché et faire baisser les prix (mettant ainsi davantage d'industries en difficulté). Le keynésianisme trouva son origine dans des situations de ce type : si les gens pouvaient avoir plus d'argent (même au prix d'un déficit budgétaire), ils achèteraient les surplus. Au lieu de détruire l'offre, il valait nettement mieux stimuler la demande.

Actuellement, tous les gouvernements ont appris, d'une façon ou d'une autre, les principes de Keynes, et l'inflation est devenue l'une des certitudes de notre temps (alors qu'au cours de presque toute la révolution industrielle, c'est la déflation qui était le phénomène le plus répandu). Mais nous avons encore toujours des surplus de production, notamment en agriculture. Les agriculteurs se délestent le long des autoroutes de leurs tomates, fruits et légumes. Cela veut-il dire que les gens n'ont pas les moyens d'acheter ces produits? Evidemment non, autrement il n'y aurait pas d'inflation.

Le problème, ce n'est pas la production, mais le coût d'accès à la production. Ces coûts sont liés au secteur tertiaire (toutes sortes de services), et ils sont dans tous les cas plus élevés que les coûts directement liés à la production : la distribution et les coûts des services déterminent l'équilibre des prix. Il est évident que la valeur économique est liée de nos jours à un système complexe qui dépend fortement des moyens d'accès à un produit (ou plutôt à ses services) : nous sommes dans une société post-industrielle, une société de service.

Les assurances ne sont bien sûr qu'une partie des services disponibles, et n'en représentent même pas la majeure partie en termes absolus. Mais elles sont très importantes et dans certains cas elles se développent plus que proportionnellement.

De plus, de toutes les activités de service, l'assurance apparaît, comme le montre l'expérience, l'activité la plus étroitement liée à la gestion des problèmes économiques dans le temps, fondés sur des hypothèses de probabilité, où il n'y aura de certitude qu'ex post.

Si l'agriculture était le point de référence de Quesnay et de beaucoup d'autres économistes avec lui, si l'industrie fut le point de référence de la théorie économique jusqu'à présent, il serait normal de penser que les économistes puissent trouver, dans l'étude de l'assurance, un excellent point de référence pour la définition et la conceptualisation de la science économique post-industrielle. Des discussions sur la notion de valeur organisées par des institutions se rattachant à la gestion du risque et à

12 A ce sujet il serait peut-être utile d'ouvrir un débat sur la notion de stocks et de flux dans la comptabilisation de la richesse. Voir [4], chap. 3 . 
l'assurance pourraient servir d'instrument à une recherche approfondie et intéressante sur ce qui, de nos jours, augmente la richesse économique et le bien-être, et sur les possibilités d'améliorer les stratégies économiques.

Concentrer la critique et la recherche sur le paradigme implicite et exclusif de la théorie économique, encore toujours étroitement liée, comme par le passé, au processus d'industrialisation, pourrait contribuer à une vision plus optimiste de ce que pourraient être la richesse et le bien-être de demain.

\section{L'avenir de l'économie : incertitude et valeur}

De nos jours, non seulement la notion d'incertitude est-elle devenue une des "réalités de la vie», liée à des perceptions spécifiques ou à un cycle économique particulier, mais depuis qu'Einstein dut implicitement admettre - contre sa volonté et sa conviction morale profonde - que «Dieu joue aux dés ", on voit apparaître une littérature de plus en plus volumineuse traitant des fondements de la nature de la science et de la structure de la connaissance. Des notions de base telles que la relativité espace/temps, l'indétermination des systemes, la relativité historique des axiomes et l'incertitude, font de plus en plus l'objet d'une étude minutieuse à la lumière des sciences naturelles et sociales ${ }^{13}$.

Contrairement à ce qui s'est passé au cours du siècle dernier, quand la science était considérée comme un moyen plus efficace d'atteindre la vérite « universelle » - et, en tant que telle, concurrente de la religion - il est aujourd'hui généralement admis que la science est un moyen de "falsification » (dans la terminologie de Karl Popper) de toutes les théories, hypothèses et événements. En d'autres termes, il n'existe pas de vérité scientifique "universelle", mais toute loi ou théorie peut être prouvée opérationnelle dans le temps et l'espace de façon limitée (ce qui signifie que "cela marche »).

Notre culture, dans l'ensemble, n'est pas encore habituée à considérer la science de cette manière. Il est révélateur de trouver, même dans des ouvrages récents, une survivance de la notion d' "objectivité universelle" de la science. Un bon exemple en est le livre d'Arthur Koestler, « Les somnambules », dans lequel la science et la religion sont considérées comme des voies complémentaires d'accès à la vérité universelle. A notre sens, sous-jacentes à ces notions de vérité et de science sont celles de temps et d'espace. Si le temps et l'espace sont considérés comme des entités pouvant être isolées à un moment ou endroit donné, ce moment « abstrait » (comme la notion d'équilibre universel de Newton) peut logiquement être considéré comme une valeur "universelle " ${ }^{14}$. Mais cette prétention à la logique «universelle » s'effondre lorsque les mathématiques nous enseignent qu'il n'existe plus d'axiomes « universels », immuables (divins).

La notion d'incertitude a, néanmoins, fait son chemin dans la pensée économique, a ce stade oủ les références au temps/espace statique demeurent encore inchangées et où le paradigme newtonien est toujours dominant. Cela s'est fait de deux façons :

13 A noter un livre très intéressant et accessible aux non-spécialistes, celui de Kline [7], ainsi qu'un court article par Little [9], qui donnent une explication claire de la vérité relative des mathématiques. Pour la physique, voir Bohm [1].

14 Pour une description et une analyse claires de ce point, voir Clark [2]. 
- la première découle de la notion de valeur subjective (voir Hicks [6]), et est reliée à l'analyse des comportements des individus avers aux risques, comme variante de la fonction d'utilité. A cet égard, la science économique devient de plus en plus une science du comportement, en concurrence avec la psychanalyse, la psychosociologie, etc. Malheureusement cette tendance n'approfondit pas l'analyse économique par rapport à l'offre: elle s'éloigne même d'une recherche empirique dont elle aurait grand besoin, sur le moment et la manière dont l'offre est générée, et sur la fonction de la technologie en tant que variable exogène.

- la seconde provient de la perception de l'incertitude comme étant due à un manque ou «asymétrie » de l'information dans un système qui, «pour des raisons de commodité », reste essentiellement statique ${ }^{15}$.

La notion de marchés incomplets résulte également de cette tentative de concevoir l'incertitude dans un paradigme cartésien.

Ces deux approches sont intéressantes, mais elles amèneront surtout, tôt ou tard, afin de pouvoir dépasser leurs capacités d'explication des faits économiques réels, à devoir surmonter l'obstacle de la notion fondamentale de valeur.

Une solution véritable découlera probablement de l'adaptation des notions de dimensions d'espace et de temps à la science économique, ce qui reviendra à tenir compte de la durée réelle et relative des phénomènes économiques. Ce processus est déjà virtuellement en marche. Il nécessitera avant tout une définition et une acceptation d'une nouvelle notion de valeur recentrée sur l'offre ${ }^{16}$.

En termes généraux, il faudrait rappeler que la vie elle-même, la vie réelle, est basée sur l'incertitude. Ce n'est pas par choix, mais en tant que condition, que le risque et l'incertitude caractérisent la vie, ne serait-ce que parce que la vie c'est le temps réel, et le risque et l'incertitude sont des éléments du temps réel. Le corollaire est que "les événements futurs - dans le paradigme d'Heisenberg - découlent d'actions et de réactions humaines imprévisibles ... alors que ... le paradigme newtonien, s'il confère à l'homme une omniscience potentielle, observateur détaché d'une réalité objective indépendante, ... soumet en même temps la société, la nature et l'homme à des lois « naturelles » qui déterminent sans équivoque possible le futur et le destin de l'homme » (voir Weisskopf [17]).

Nous insistons sur ce point: de tels principes, au cours de la révolution industrielle classique, ont été assez efficaces. Mais notre siècle a assisté à la dégradation de leurs conséquences, y compris la lente destruction de la notion de responsabilité et de liberté d'action.

Un économiste, René Passet, a écrit récemment un ouvrage [12] dans lequel il démontre que l'économie a traité jusqu'à présent des « objets morts". Il commence son analyse en attirant l'attention sur la correspondance entre la pensée économique au cours

15 Voir Diamond et Rothschild [3], chapitres suivants : "Individual choice in a static setting », "General equilibrium in a static setting», "Sequential choice and equilibrium with limited information $»$.

${ }_{16}$ C'est précisément ce type de dialogue que le Rapport sur la Richesse et le Bien-Etre [4] s'est efforcé de développer. 
des deux derniers siecles et les changements survenus dans la notion de science ${ }^{17}$. L'opposition qu'il relève entre ce qui est mort et ce qui est vivant est analoque à l'opposition entre temps statique et temps réel. La transition entre l'un et l'autre est la transition entre la certitude utopique et le défi de l'incertitude réelle, entre une pensée essentiellement déterministe et la possibilité d'édifier une véritable responsabilité et liberté, en se fondant sur un monde largement indéterminé.

Il est évident que trop d'incertitude conduit à l'impuissance, et c'est précisément la raison pour laquelle ses origines doivent être définies chaque fois que possible. Mais en fin de compte, le probleme est de savoir comment vivre mieux, et ceci en faisant mieux face au risque.

La grande incertitude qui règne actuellement dans le monde contemporain pourrait engendrer, chez certains, le désespoir. Mais au niveau de l'action, elle représente un merveilleux défi à la culture, à la science, et à la théorie économique.

\section{RÉFÉRENCES}

1. BOHM, D. : Wholeness and the Implicate Order, Routledge \& Keegan, London, 1980.

2. CLARK, R. W. : Einstein, the Life and Times, Hodder \& Stoughton, London, 1973.

3. DIAMOND, P., and ROTHSCHILD, M.: Uncertainty in Economics, Academic Press, New York, 1978.

4. GIARINI, O.: Dialogue on Wealth and Welfare, Pergamon Press, Oxford, 1980. Version française, Economica, Paris, 1981.

5. GIARINI, O., and LOUBERGÉ, H. : The Diminishing Returns of Technology, Pergamon Press, Oxford, 1978. Version française, Dunod, Paris, 1979.

6. HICKS, J. R. : Value and Capital, Oxford University Press, 1939.

7. KLINE, M. : Mathematics - The Loss of Certainty, Oxford University Press, 1980.

8. ILLICH, I. : «Shadow-Work », paper presented at a conference in Kassel, September 1980.

9. LITTLE, J.: «The uncertain craft of mathematics», New Scientist, 88 (4 December 1980), 626-628.

10. MARSHALL, A.: Principles of Economics, MacMillan, London, 1977 (eighth edition reprinted).

11. MAYER, T. : «Economics as a hard science: Realistic goal or wishful thinking?", Economic Inquiry, 18 (April 1980), 165-178.

12. PASSET, R. : L'Economique et le Vivant, Payot, Paris, 1979.

13. PFEFFER, I.: Insurance and Economic Theory, Irwin, Homewood (Illinois), 1956.

17 Sa vérification du paradigme économique sur la base des travaux d'llia Prigogine s'avère prometteuse. 
14. PFEFFER, 1., and KLOCK, D. R. : Perspectives on Insurance, Prenctice Hall, Englewood Cliffs (New Jersey), 1974.

15. PRIGOGINE, 1., and STENGERS, 1. : La Nouvelle Alliance, Gallimard, Paris, 1979. Voir aussi : PRIGOGINE, 1.: Between Time and Eternity - Nehru and Einstein, Jawahardal Nehru Lecture, 1983, polycopié.

16. SMITH, A. : The Wealth of Nations, Penguin Books, Hammondsworth (Middlesex), 1977 (first published in 1776).

17. WEISSKOPF, W. A. : "The method is the ideology: From a Newtonian to a Heisenbergian paradigm in economics ", Journal of Economic Issues, 13 (December 1979), 868-884. 